\title{
Renal Subcapsular Hematoma Formation Due to Hydronephrosis Caused by Recurrent Uterine Cervical Cancer
}

\author{
Hana Nakamura ${ }^{\mathrm{a}, \mathrm{c}}$, Kazuyoshi Ko ${ }^{\mathrm{b}}$, Hisami Kiseki ${ }^{\mathrm{a}}$, Shun-ichi Ikeda ${ }^{\mathrm{a}, \mathrm{c}, \mathrm{d}}$
}

\begin{abstract}
Most non-traumatic renal subcapsular hematomas are found in the presence of primary or metastatic renal tumors, or in the presence of vascular disease of the renal blood vessels. We managed an asymptomatic renal subcapsular hematoma that formed due to uterine cervical cancer that metastasized to the left common iliac lymph nodes. A 48-year-old woman with stage IB1 cervical cancer underwent neoadjuvant chemotherapy and concurrent chemoradiation following a radical hysterectomy. Six months after the completion of her first treatment, she developed left-sided hydronephrosis, a left subcapsular hematoma and left common iliac lymph nodes enlargement as demonstrated with contrast-enhanced computed tomography. Although a renal subcapsular hematoma is rarely a symptom of cervical cancer recurrence, it should be considered if other neoplastic or vascular diseases are ruled out.
\end{abstract}

Keywords: Renal subcapsular hematoma; Uterine cervical cancer; Hydronephrosis

\section{Introduction}

Renal subcapsular hematomas are commonly the result of trauma. However, they can also be caused by non-traumatic etiologies, such as the presence of primary or metastatic renal tumors or vascular disease of the renal vessels [1]. Here we describe an atraumatic and asymptomatic renal subcapsular hematoma that formed due to cervical cancer metastasis to the left common iliac lymph nodes.

Manuscript submitted December 20, 2020, accepted January 4, 2021

Published online January 19, 2020

aDepartment of Obstetrics and Gynecology, Kohseichuo General Hospital, Tokyo, Japan

bepartment of Urology, Kohseichuo General Hospital, Tokyo, Japan

'Both authors contributed equally to this manuscript.

${ }^{\mathrm{d} C o r r e s p o n d i n g ~ A u t h o r: ~ S h u n-i c h i ~ I k e d a, ~ D e p a r t m e n t ~ o f ~ O b s t e t r i c s ~ a n d ~ G y n e-~}$ cology, Kohseichuo General Hospital, 1-11-7, Mita, Meguro-ku, Tokyo 1538581, Japan. Email: shiked@kohseichuo.jp

doi: https://doi.org/10.14740/jmc3640

\section{Case Report}

A 48-year-old woman with uterine cervical cancer was referred to the gynecology department of our hospital. A pelvic examination revealed a tumor with a diameter of $3.0 \mathrm{~cm}$ in her cervix without bilateral parametrial involvement. Contrast-enhanced computed tomography (CECT) was negative for swelling of the pelvic lymph nodes. Histopathologic examination of the cervix revealed squamous cell carcinoma of the cervix, and International Federation of Gynecology and Obstetrics (FIGO) stage IB1 disease was diagnosed. The squamous cell carcinoma antigen (SCC-ag) level was $9.6 \mathrm{ng} / \mathrm{mL}$. We proposed a radical hysterectomy (RH) or concurrent chemoradiation (CCRT). However, she requested RH following neoadjuvant chemotherapy (NAC). Three courses of TP therapy (paclitaxel $135 \mathrm{mg} / \mathrm{m}^{2}$ and cisplatin $50 \mathrm{mg} / \mathrm{m}^{2}$ ) were performed. However, the cervical lesion was $28 \mathrm{~mm}$, representing only a slightly decreased size compared with pre-treatment measurements. An RH with bilateral salpingo-oophorectomy (BSO) was performed. Surgical pathology showed mass infiltration into the left parametrium. There were no metastases to the pelvic lymph nodes. As the patient was pT2bN0, CCRT $\left(40 \mathrm{mg} / \mathrm{m}^{2} /\right.$ week, Liniac 1.8 Gy $\times 25$ fractions with the upper end of the irradiation field at L5) was performed postoperatively. The SCC-ag level after CCRT was $1.4 \mathrm{ng} / \mathrm{mL}$. At regular examinations 6 months after the end of CCRT, the SCC-ag level increased to $3.2 \mathrm{ng} / \mathrm{mL}$. A repeat CECT revealed left hydronephrosis, a left subcapsular hematoma and left common iliac lymph node swelling (Figs. 1 and 2). The patient had no back pain, no abdominal pain, no fever and no hypertension. A double-J stent was placed into the left urethra under cystoscopic guidance. A field from the left common iliac lymph nodes to the left para-aortic lymph nodes was irradiated with $60 \mathrm{~Gy}$. Follow-up ultrasound image after radiotherapy showed that the hematoma was reduced to less than three quarters of its initial size.

\section{Discussion}

Here, we present a case of the asymptomatic formation of a renal subcapsular hematoma in the setting of metastatic uterine cervical cancer to the lymph nodes. Our experience indicated the following two important clinical points. First, hydronephrosis caused by ureteral stenosis due to recurrent cervical 


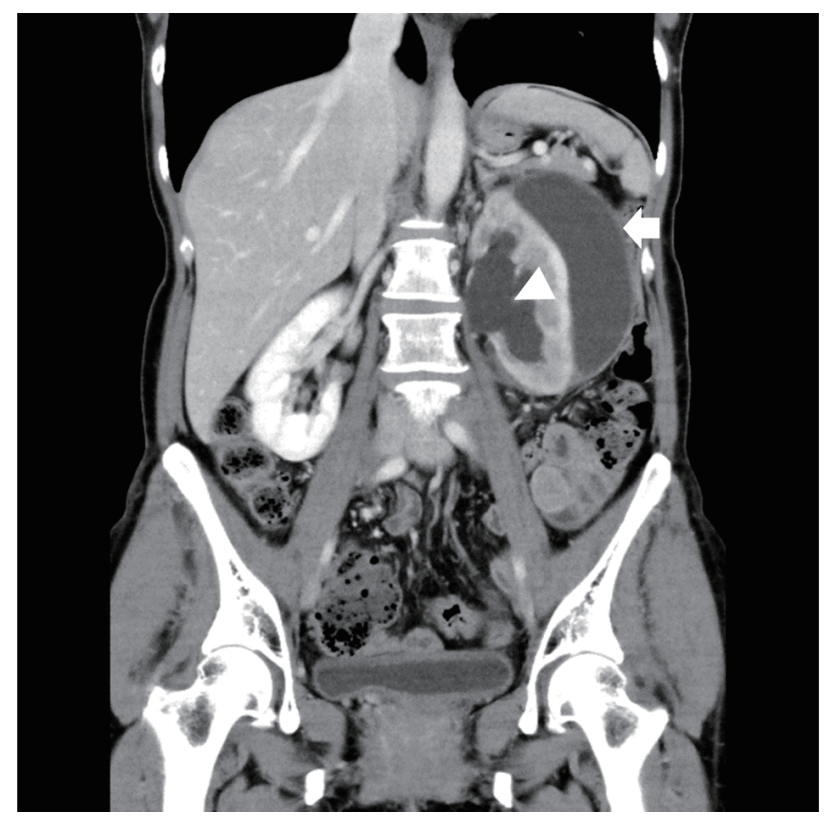

Figure 1. A coronal view on CECT scan revealed a low attenuation fluid collection in the subcapsular area (arrow) in the setting of dilation of the renal pelvis (arrowhead). CECT: contrast-enhanced computed tomography.

cancer in the left common iliac lymph nodes resulted in a subcapsular hematoma. Second, our patient had no symptoms even after the formation of the hematoma.

First, our experience showed that hydronephrosis from ureteral stenosis due to cervical cancer metastatic to the left common iliac lymph nodes formed a subcapsular hematoma. Kawashima et al [1] in their review classify subcapsular hematomas as traumatic or non-traumatic, and it is known that most subcapsular hematomas are traumatic. Zhang et al [2] estimated in their meta-analysis that $61.5 \%$ of non-traumatic subcapsular hematomas were the result of tumors, $17 \%$ were due to vascular disease, $2.4 \%$ were from infections and $6.5 \%$ were iatrogenic. The neoplastic breakdown was $31.5 \%$ malignant and $29.7 \%$ benign. Both primary and metastatic tumors were present, and tumors demonstrated a high degree of vascularity histologically.

The patient's subcapsular hematoma was not the direct result of tumor invasion or metastasis into the kidney. Ureter stenosis due to lymph node metastases caused the patient's hydronephrosis, which led to her subcapsular hematoma. To the best our knowledge, this is the first report of a subcapsular hematoma formed by hydronephrosis due to ureteral stenosis from lymph node metastases. Cervical cancer infiltration often causes hydronephrosis. In the case of slow-growing cancers, hydronephrosis caused by infiltration of the urethral system may progress to a nonfunctioning kidney. It has been reported that a giant hydronephrosis is sometimes mistaken for a neoplasm [3]. In contrast, when a high load is rapidly generated in the urethral system due to urinary tract stones or rapid diuresis, the upper urethral system will rupture [4]. Based on these facts, the development of the subcapsular hematoma from hydronephrosis in the present case was thought to occur from subacute progression, not acute or chronic events.

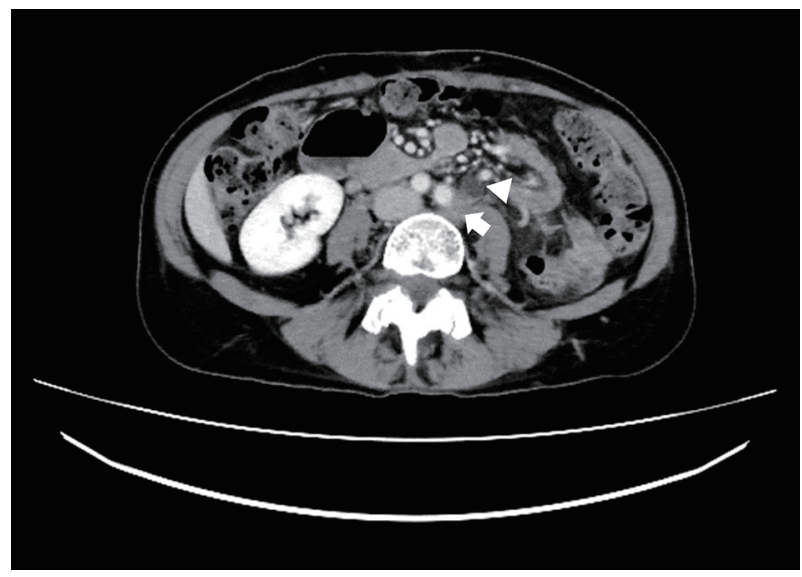

Figure 2. A transverse view on CECT scan showed left common iliac lymph node swelling (arrow) and a dilated left ureter (arrowhead). CECT: contrast-enhanced computed tomography.

Based on our knowledge that subcapsular hematomas form as the result of vascular rupture, we have identified three mechanisms by which hydronephrosis can cause a subcapsular hematoma. One is the result of post-transurethral ureteroscope lithotripsy (URSL) [5]. When the renal calyx is dilated due to stones, sudden dilation of the intrarenal pressure by URSL can lead to the rupture of blood vessels around the kidney and within the renal parenchyma, leading to the formation of a subcapsular hematoma. This mechanism is similar to how hematuria is caused by acute urinary retention [6]. Submucosal bladder wall veins that are rapidly attenuated and compressed from the sudden dilation of the bladder result in bleeding and hematuria. The second mechanism is based on animal experimental data that demonstrate that ligation of the renal veins has been shown to result in severe interstitial bleeding, vascular congestion and subcapsular hematoma after $90 \mathrm{~min}$ [7]. The third is pregnancy-related varix formation. A varix is a common complication of pregnancy that is formed by pregnancyinduced changes to the uterus and hormones that results in the compression of the inferior vena cava (IVC) and pelvic veins, leading to bladder varicose veins and varix rupture that can result in to frank blood in the urine during pregnancy [8].

In our case, the first of the proposed mechanisms did not occur because the intrarenal pressure was not released. The second proposed mechanism also did not occur because renal pelvis dilation-induced renal venous blood flow did not develop. In our case, the renal subcapsular hematoma is thought to be formed by renal pelvis dilation that led the kidney vessels to dilate, which probably contributed to varix formation and subcapsular hematoma.

Our second clinical point is that our patient developed an asymptomatic subcapsular hematoma. Daskalopoulos et al [9] showed that all 13 subcapsular hematomas presented in their work presented along with flank or abdominal pain. The breakdown of their cases was hypervascularity around a renal tumor in nine cases (angiolipoma: five cases; renal cell carcinoma: four cases), three cases of vascular disease and one case due to anticoagulation. Manno et al [10] performed a prospective analysis of 471 patients who underwent a renal biopsy and re- 
ported a post-biopsy hematoma rate of $33.3 \%$ of which $90.4 \%$ were clinically asymptomatic. These results suggest that the appearance of symptoms depends on the size of the hematoma and its rate of formation. That is, the risk of bleeding from a renal biopsy is low because the biopsy is performed under ultrasound guidance, avoiding the veins and arteries.

Bleeding from underlying diseases such as hypervascular tumors is thought to be mostly arterial from tumor vessels.

Hematoma formation is rapid when an artery ruptures, and symptoms due to pressure on the surrounding tissues are likely to occur. Furthermore, it is conceivable that high blood flow pressure leads to bleeding beyond the capsule. Therefore, in the case of arterial bleeding, it is easily presumed that severe symptoms are likely to occur. Bleeding from veins including a varix takes time to form a hematoma, and it is expected that capsular rupture is less likely to occur due to low pressure and the manifestation of symptoms is uncommon. In our case, we speculated that hydronephrosis caused dilation of the veins around the kidneys that ruptured. However, it was thought that the subcapsular hematoma was asymptomatic because of its slow rate of formation.

It should be noted that complications from a subcapsular hematoma include hypertension and infection. Hypertension is thought to be caused by compression of the renal parenchyma by the hematoma, also known as a Page kidney [11]. Compression of the renal parenchyma reduces renal blood flow and activates the renin-angiotensin-aldosterone system, resulting in hypertension and treatment resistance. Delayed intervention may require a nephrectomy. Hematoma can also be a source of infection. Furthermore, when hydronephrosis is the cause of a renal subcapsular hematoma, as in this case, urinary stasis is likely to cause bacteriuria and increase the chance of infecting the hematoma.

\section{Conclusion}

Cervical cancer recurrence in the lymph nodes caused hydronephrosis by excluding the ureter, which resulted in the development of a subcapsular hematoma. Since no symptoms suggestive of a subcapsular hematoma, including pain, developed, it is presumed that the hematoma was venous in origin and formed slowly. Although a renal subcapsular hematoma is rarely a symptom of cervical cancer recurrence, cancer recurrence should be considered in the setting of subcapsular hematomas until neoplastic or vascular diseases are ruled out. When following up on subcapsular hematomas, attention should be paid to the development of infections and hypertension.

\section{Acknowledgments}

None to declare.

\section{Financial Disclosure}

None to declare.

\section{Conflict of Interest}

None to declare.

\section{Informed Consent}

Informed consent was obtained from the patient prior to completing the case report.

\section{Author Contributions}

$\mathrm{HN}$ and SiI contributed to the writing of the manuscript. HN, $\mathrm{KK}, \mathrm{HK}$ and SiI reviewed and adjusted the manuscript prior to submission.

\section{Data Availability}

The data supporting the findings of this study are available from the corresponding author upon reasonable request.

\section{References}

1. Kawashima A, Sandler CM, Corl FM, West OC, Tamm EP, Fishman EK, Goldman SM. Imaging of renal trauma: a comprehensive review. Radiographics. 2001;21(3):557574.

2. Zhang JQ, Fielding JR, Zou KH. Etiology of spontaneous perirenal hemorrhage: a meta-analysis. J Urol. 2002;167(4):1593-1596.

3. Schrader AJ, Anderer G, von Knobloch R, Heidenreich A, Hofmann R. Giant hydronephrosis mimicking progressive malignancy. BMC Urol. 2003;3:4.

4. Ikeda S, Ishikawa M, Kato T. Spontaneous ureteral rupture during concurrent chemoradiotherapy in a woman with uterine cervical cancer. Gynecol Oncol Rep. 2015;13:18-19.

5. Zhang P, Hu WL. Sudden onset of a huge subcapsular renal hematoma following minimally invasive ureteroscopic holmium laser lithotripsy: A case report. Exp Ther Med. 2015;10(1):335-337.

6. Naranji I, Bolgeri M. Significant upper urinary tract hematuria as a rare complication of high-pressure chronic retention of urine following decompression: a case report. J Med Case Rep. 2012;6:254.

7. Liu X, Li N, Xu T, Sun F, Li R, Gao Q, Chen L, et al. Effect of renal perfusion and structural heterogeneity on shear wave elastography of the kidney: an in vivo and ex vivo study. BMC Nephrol. 2017;18(1):265.

8. Hallamore SL, Grills RJ, Neerhut G, Lawrentschuk N. Submucosal vesical varicosities causing hematuria and retention of urine in pregnancy: cystovarix. Am J Obstet Gynecol. 2007;196(5):e29-30.

9. Daskalopoulos G, Karyotis I, Heretis I, Anezinis P, Mavromanolakis E, Delakas D. Spontaneous perirenal hem- 
orrhage: a 10-year experience at our institution. Int Urol Nephrol. 2004;36(1):15-19.

10. Manno C, Strippoli GF, Arnesano L, Bonifati C, Campobasso N, Gesualdo L, Schena FP. Predictors of bleed- ing complications in percutaneous ultrasound-guided renal biopsy. Kidney Int. 2004;66(4):1570-1577.

11. Whitworth PW, 3rd, Dyer RB. The "Page kidney". Abdom Radiol (NY). 2017;42(9):2387-2388. 